Anaesthesist 2019.68:341-342

https://doi.org/10.1007/s00101-019-0604-0

(c) Springer Medizin Verlag $\mathrm{GmbH}$, ein Teil von Springer Nature 2019

\title{
P. Meybohm
}

Klinik für Anästhesiologie, Intensivmedizin und Schmerztherapie (KAIS), Universitätsklinikum Frankfurt, Frankfurt am Main, Deutschland

\section{Versorgungsforschung - Experimentierst Du noch oder forschst du schon?}

Nachdem in den letzten Jahrzehnten der Fokus der Gesundheitsforschung (und der Vergabe der Forschungsmittel) v. a. auf molekularbiologischer Grundlagenforschung und krankheitsorientierter (tierexperimenteller) Forschung im Labor lag und weiterliegt, rücken die klinische Forschung und die Versorgungsforschung zunehmend in den Mittelpunkt.

Hierbei stehen klinische Forschung (randomisierte kontrollierte Studien) sowie Versorgungsforschung komplementär zueinander.

Während in klinischen Studien die Wirksamkeit neuer Behandlungen bei ausgewählten Patienten geprüft wird und die Forschung damit auf das Verständnis kausaler Mechanismen und auf die Wirksamkeit von neuen Therapien unter idealen Studienbedingungen abzielt, bleibt letztendlich unklar, ob neue Therapieverfahren den Patienten flächendeckend im Alltag tatsächlich nützen.

Zwar können wissenschaftlich fundierte Behandlungsempfehlungen und Leitlinien bei der Umsetzung helfen, doch hängt eine erfolgreiche Implementierung von vielen anderen Faktoren ab. Diese sind beispielsweise die Therapietreue des Patienten, die jeweilige Lebenswelt des Patienten, Kosten der jeweiligen Produkte oder Medikamente, Fort- und Weiterbildung von Ärzten und Pflegekräften, die Art und Intensität der intersektoralen Kooperation u.v.a.

Die Versorgungsforschung untersucht nun genau diese „Wirklichkeit“. Die Versorgungsforschung prüft Hypothesen zur Wirksamkeit von gesundheitsrelevanten
Ansätzen unter Alltagsbedingungen und im Hinblick auf deren Effizienz in der realen Welt. Dabei kann die klinische Wirksamkeit unter Alltagsbedingungen jedoch stärker oder schwächer ausfallen. Neben den Analysen zu Über-, Unter- und Fehlversorgung werden zudem die individuelle Patientenperspektive sowie die Gesundheitsökonomie berücksichtigt.

Vor diesem Hintergrund überrascht es kaum, dass die Versorgungsforschung aktuell viel Aufwind und einen Höhenflug erlebt. Forschungsgelder von mehreren Hundert Millionen Euro für Hunderte Projekte wurden in den vergangenen Jahren ausgeschrieben, $u$. a. von dem Bundesministerium für Bildung und Forschung (BMBF) [1], dem Bundesgesundheitsministerium (BMG), der Deutschen Forschungsgemeinschaft (DFG) [2], der Bundesärztekammer (BÄK), dem Innovationsfond [3], den Bundesländern [4], Krankenkassen, Stiftungen etc.

Der aktuelle Beitrag von Blecha et al. gibt am Beispiel des akuten Lungenversagens einen Überblick über den aktuellen Stand der intensivmedizinischen Versorgungsforschung in Deutschland.

Im Fachgebiet AINS werden inzwischen allein durch den Innovationsfond zahlreiche Projekte in Millionenhöhe ge-

fördert, z. B.

- Teleintensivmedizin Nordrhein-

Westfalen (Telnet@NRW),

- Enhanced Recovery after Intensive Care (ERIC),

- Schmerz: Patientenorientiert. Abgestuft. Interdisziplinär. Netzwerk (PAIN 2020), 
- Prähabilitation von älteren Patienten mit Gebrechlichkeitssyndrom vor elektiven Operationen (PRÄP-GO),

- Validierung und Optimierung der Nutzbarkeit von Routinedaten zur Qualitätsverbesserung des SepsisManagements im Krankenhaus (OPTIMISE),

- Patientensicherheit, Wirtschaftlichkeit und Lebensqualität: Reduktion von Delirrisiko und postoperativer kognitiver Dysfunktion (POCD) nach Elektivoperationen im Alter (PAWEL),

- Optimierung der perioperativen Versorgung älterer Patienten (PeriAge),

- Folgeschäden nach prolongierter Intensivbehandlung: Entwicklung und Pilotierung einer IntensivNachsorgeambulanz (PINA) [3].

An dieser Stelle darf den in diesem hochkompetitiven Bereich erfolgreichen Projektleitern und Antragstellern gratuliert werden.

In Anbetracht der zahlreichen spannenden wissenschaftlichen Fragestellungen zum Thema Versorgungsalltag AINS möchte ich zugleich aber auch alle Leser ermutigen, die Herausforderung anzunehmen und das Thema Versorgungsforschung in AINS zu stärken.

\section{Korrespondenzadresse}

Univ.-Prof. Dr. med. P. Meybohm, MHBA

Klinik für Anästhesiologie, Intensivmedizin und Schmerztherapie (KAIS), Universitätsklinikum Frankfurt

Theodor-Stern-Kai 7, 60590 Frankfurt am Main, Deutschland

patrick.meybohm@kgu.de

Interessenkonflikt. P. Meybohm gibt an, dass kein Interessenkonflikt besteht.

\section{Literatur}

1. Bundesministerium für Bildung und Forschung (BMBF) (2014) Versorgungsforschung - nah am medizinischen Alltag. https://www.bmbf.de/de/ versorgungsforschung-nah-am-medizinischenalltag-392.html.Zugegriffen:22. Apr. 2019

2. Deutsche Forschungsgemeinschaft (DFG) (2014) Nachwuchsakademien Versorgungsforschung. https://www.dfg.de/foerderung/grundlagen_ rahmenbedingungen/fachwissenschaften/ lebenswissenschaften/themen/versorgungsfor schung/nachwuchsakademien/index.html. Zugegriffen:22.Apr. 2019
3. Innovationsausschuss des Gemeinsamen Bundesausschuss (2019) Geförderte Projekte. https:// innovationsfonds.g-ba.de/projekte/.Zugegriffen: 22. Apr. 2019

4. Bayerisches Landesamt für Gesundheit und Lebensmittelsicherheit (2019) Projekte der Versorgungsforschung. https://www.lgl. bayern.de/gesundheit/gesundheitsversorgung/ informationsplattform_versorgungsforschung/ projekte/index.htm.Zugegriffen:22. Apr. 2019
Auch online Zugang zu allen Beiträgen von Der Anaesthesist

Wussten Sie, dass Sie als Abonnent dieser Zeitschrift automatisch online Zugriffsrechte auf das gesamte Beitragsarchiv haben?

Werden Sie Abonnent von Der Anaesthesist und erhalten Sie zwölf mal im Jahr Ihre Ausgabe nach Hause geliefert. Der Zugang zur gesamten Online-Bibliothek ist im Abonnement der Zeitschrift inbegriffen. Für den Zugang müssen Sie sich lediglich einmal über www.springermedizin.de/register registrieren.

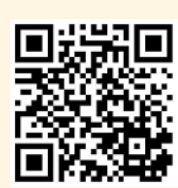

Über diesen QR-Code schnel und einfach registrieren

Bitte nutzen Sie für die Registrierung Vorund Nachname und Lieferadresse wie beim Abonnement der Zeitschrift (siehe Adressaufkleber auf Ihrem Heft). So kann im System die Zugehörigkeit zu Ihrer Zeitschrift sichergestellt werden.

Aufgrund des Heilmittelwerbegesetzes dürfen die Inhalte der Website nur medizinischen Fachkreisen zur Verfügung gestellt werden. Bei der Anmeldung bitten wir Sie deshalb einen Berufsnachweis vorzulegen.

Bei Medizinern mit Mitgliedschaft in der deutschen Ärztekammer reicht die einheitliche Fortbildungsnummer (EFN). Alternativ schicken Sie eine Bestätigung des Arbeitgebers, Studiennachweis oder andere Zeugnisse ganz unkompliziert an kundenservice@springermedizin.de.

Mit Benutzername und Passwort haben Sie außerdem Zugang zu den freien Inhalten auf den Seiten von: https://www.springermedizin.de/ https://www.aerztezeitung.de/

Sollten Fragen oder Probleme auftauchen, wenden Sie sich einfach an Ihren Kundenservice:

kundenservice@springermedizin.de 